\title{
FREE BOUNDARY REGULARITY FOR THE POISSON KERNEL BELOW THE CONTINUOUS THRESHOLD
}

\author{
Carlos E. Kenig and Tatiana Toro
}

\section{Introduction}

The purpose of this note is to announce the proof of the conjecture stated by the authors in [KT1]. In [KT2] we show that the "weak" regularity of the Poisson kernel of a domain fully determines the geometry of its boundary. Namely, we show that if $\Omega$ is a $\delta$-Reifenberg flat chord arc domain for $\delta>0$ small enough, and the logarithm of the Poisson kernel has vanishing mean oscillation, then the unit normal vector to the boundary also has vanishing mean oscillation.

Jerison (see $[\mathrm{J}]$ ) introduced the end point problem in higher dimensions, but treated it under more restrictive assumptions, namely that the boundary is given locally as a Lipschitz graph, and the normal derivative data is continuous as opposed to having vanishing mean oscillation. His paper is based on the work of Jerison-Kenig [JK2] and first points out the connection with the work of Alt and Caffarelli $[\mathrm{AC}]$. There is an error in Lemma 4 of Jerison's paper. Nevertheless in our previous work (see [KT1]) we made considerable use of the ideas in $[\mathrm{J}]$. In the present work we bypass this approach.

It is interesting to compare our results with those of Pommerenke $[\mathrm{P}]$ and those of Alt and Caffarelli $[\mathrm{AC}]$. In all cases the oscillation of the logarithm of the Poisson kernel, $k_{A}$, controls the geometry of the boundary and the oscillation of the unit normal.

Main Theorem. Assume that

1. $\Omega \subset \mathbb{R}^{n+1}$ is a $\delta$-Reifenberg flat chord arc domain for some $\delta>0$ small enough.

2. $\log k_{A} \in \operatorname{VMO}(\partial \Omega)$,

Then $\Omega$ is a chord arc domain with vanishing constant, i.e $\vec{n} \in \operatorname{VMO}(\partial \Omega)$.

Remark. Note that in [KT3] we have shown the converse of this, namely that if $\Omega \subset \mathbb{R}^{n+1}$ is a $\delta$-Reifenberg flat chord arc domain and $\vec{n} \in \operatorname{VMO}(d \sigma)$ then $\log k_{A} \in \operatorname{VMO}(d \sigma)$.

The results of Pommerenke and Alt and Caffarelli are as follows.

Received July 25, 2001.

The authors were partially supported by the NSF. 
Theorem $[\mathrm{P}]$ Let $\Omega \subset \mathbb{R}^{2}$ be a chord arc domain. Then $\Omega$ is a chord arc domain with vanishing constant if and only if $\log k_{A} \in \mathrm{VMO}(\partial \Omega)$.

Theorem $[\mathrm{AC}]$ Assume that

1. $\Omega \subset \mathbb{R}^{n+1}$ is a $\delta$-Reifenberg flat chord arc domain for some $\delta>0$ small enough;

2. $\log k_{A} \in C^{0, \beta}$ for some $\beta \in(0,1)$.

Then $\Omega$ is a $C^{1, \alpha}$ domain for some $\alpha \in(0,1)$ which depends on $\beta$ and $n$.

Recall that a set of locally finite perimeter (see [EG]) $\Omega \subset \mathbb{R}^{n+1}$ is said to be a $\delta$-Reifenberg flat chord arc domain, if $\Omega$ is a $\delta$-Reifenberg flat domain whose boundary is Ahlfors regular (i.e. $\mathcal{H}^{n}(\partial \Omega \cap B(Q, r)) \sim r^{n}$ for $Q \in \partial \Omega$ and $r \in(0$, diam $\Omega)$ ). Recall also that a $\delta$-Reifenberg flat domain in $\mathbb{R}^{n+1}$ is a domain satisfying the separation property, and such that its boundary can be locally approximated by $n$-dimensional affine spaces in the Hausdorff distance sense ( $\delta$ measures how accurate this approximation is). Here $\delta>0$ is chosen so that a $\delta$-Reifenberg flat domain is NTA (see [JK1] and [KT3]). Recall also that a set of locally finite perimeter whose boundary is Ahlfors regular is said to be a chord arc domain if it an NTA domain.

We would like to emphasize that the hypothesis 1 above is necessary. Keldysh and Lavrentiev (see [KL]) constructed a domain in $\mathbb{R}^{2}$ whose boundary is rectifiable but not Ahlfors regular, whose Poisson kernel is identically equal to 1 and which is not $C^{1}$. Moreover there are examples of domains in $\mathbb{R}^{2}$ whose boundary is Reifenberg flat with vanishing constant, rectifiable but not Ahlfors regular, for which the logarithm of the Poisson kernel is Hölder continuous and which are not $C^{1}$ domains (see [Du]). Furthermore, if $n \geq 2$ Pommerenke's result does not hold. Namely there are examples of chord arc domains whose Poisson kernel has logarithm in VMO, but whose boundaries contain a neighborhood of the vertex of a double cone (see $[\mathrm{AC}]$ and $[\mathrm{KT} 1]$ ).

The basic technique used to prove the Main Theorem is a blow up argument which uses geometric and analytic information about the free boundary regularity problem for the Poisson kernel. We combine this with a generalized version of the Alt-Caffarelli result (see Appendix B in [KT2]) to show that given any blow up sequence there is a subsequence such that (modulo rotation and translation) the blow-up domains converge to the upper half space $\mathbb{R}_{+}^{n+1}$ and the blow-up functions converge to the function $u\left(\bar{x}, x_{n+1}\right)=x_{n+1}$.

\section{AC type result}

The following result establishes a criteria, via potential theory, for a Reifenberg flat chord arc domain to be a half space. This criteria is solely stated in terms of the behavior of the Poisson kernel with pole at infinity. Theorem 2.1 and Corollary 2.1, whose proofs appear in [KT4], are versions of the results in Sections 7 and 8 in $[\mathrm{AC}]$. 
Theorem 2.1. There exists $\delta_{n}>0$ so that if $\Omega \subset \mathbb{R}^{n+1}$ is an unbounded $\delta$ Reifenberg flat chord arc domain for $\delta \in\left(0, \delta_{n}\right)$ and $u$ and $h$ satisfy

$$
\begin{cases}\Delta u=0 & \text { in } \Omega \\ u>0 & \text { in } \Omega \\ u=0 & \text { on } \partial \Omega\end{cases}
$$

and

$$
\int_{\Omega} u \Delta \varphi=\int_{\partial \Omega} \varphi h d \sigma \text { for all } \varphi \in C_{c}^{\infty}\left(\mathbb{R}^{n+1}\right)
$$

with

$$
\sup _{X \in \Omega}|\nabla u(X)| \leq 1 \text { and } h(Q) \geq 1 \quad \mathcal{H}^{n} \text { a.e. } Q \in \partial \Omega,
$$

then $\Omega$ is a half space, and in suitable orthogonal coordinates $u\left(\bar{x}, x_{n+1}\right)=x_{n+1}$.

In Theorem $2.1 u$ denotes the Green's function with pole at infinity and $h=\frac{d \omega}{d \sigma}$ the corresponding Poisson kernel. Here $\omega$ denotes the harmonic measure with pole at infinity, and $\sigma=\mathcal{H}^{n}\llcorner\partial \Omega$. If $\Omega$ is a half space Liouville's theorem and $(2.3)$ ensure that $u\left(\bar{x}, x_{n+1}\right)=x_{n+1}$ (see [KT3]).

Corollary 2.1. There exists $\delta_{n}>0$ so that for $\delta \in\left(0, \delta_{n}\right)$, if $\Omega \subset \mathbb{R}^{n+1}$ is an unbounded $\delta$-Reifenberg flat chord arc domain, $u$ and $h$ satisfy (2.1), (2.2) and

$$
h=1 \mathcal{H}^{n}-\text { a.e on } \partial \Omega
$$

then $\Omega$ is a half space.

Corollary 2.1 follows from Theorem 2.1 and the following estimate for the gradient of the Green's function (see [KT2], Section 3).

Theorem 2.2. There exists $\delta_{n}>0$ so that for $\delta \in\left(0, \delta_{n}\right)$, if $\Omega \subset \mathbb{R}^{n+1}$ is an unbounded $\delta$-Reifenberg flat chord arc domain, and $u$ and $h$ satisfy (2.1), (2.2) with $\log h \in \operatorname{VMO}(\partial \Omega)$ then for all $X \in \Omega$ we have

$$
|\nabla u(X)| \leq \int_{\partial \Omega} h(Q) d \omega^{X}(Q) .
$$

\section{Blow up technique}

We present a general construction of blow-up sequences for Reifenberg flat chord arc domains whose Poisson kernel has logarithm in VMO. The main result is that any such sequence has a subsequence whose limit satisfies the hypothesis of the generalized version of Alt's and Caffarelli's result presented in $\S 2$. For the proofs we refer the reader to $\S 4$ in [KT2].

Let $\Omega \subset \mathbb{R}^{n+1}$ be a $\delta$-Reifenberg flat chord arc domain, with $\delta>0$ small enough. Here $u$ denotes either the Green's function with pole at $A$ or with pole at infinity, $\omega$ and $h$ denote the corresponding harmonic measure, and Poisson kernel respectively, and $d \omega=h d \sigma$ where $\sigma=\mathcal{H}^{n}\llcorner\partial \Omega$ (see definitions in [KT1]). We assume that $\log h \in \operatorname{VMO}(\partial \Omega)$. Let $Q_{i} \in \partial \Omega$, and assume $Q_{i} \rightarrow Q_{\infty} \in \partial \Omega$ 
as $i \rightarrow \infty$. Let $\left\{r_{i}\right\}_{i \geq 1}$ be a sequence of positive numbers so that $\lim _{i \rightarrow \infty} r_{i}=0$. Consider the domains

$$
\Omega_{i}=\frac{1}{r_{i}}\left(\Omega-Q_{i}\right) \text { with } \partial \Omega_{i}=\frac{1}{r_{i}}\left(\partial \Omega-Q_{i}\right) .
$$

Consider also the functions $u_{i}$ on $\Omega_{i}$ defined by

$$
u_{i}(Z)=\frac{u\left(r_{i} Z+Q_{i}\right)}{r_{i} \oint_{B\left(Q_{i}, r_{i}\right)} h d \sigma} .
$$

Note that

$$
\Delta u_{i}=0 \text { on } \Omega_{i}^{*} \subset \Omega_{i},\left.\quad u_{i}\right|_{\partial \Omega_{i}}=0
$$

and

$$
d \omega_{i}(Q)=h_{i}(Q) d \sigma_{i}(Q) \text { for } \mathcal{H}^{n} \text {-a.e. } Q \in \partial \Omega_{i},
$$

where $\Omega_{i}^{*}=\Omega_{i}$ if $u$ is the Green's function with pole at infinity and $\Omega_{i}^{*}=$ $\Omega_{i} \backslash\left\{\frac{A-Q_{i}}{r_{i}}\right\}$, if $u$ is the Green's function with pole at $A$. Here $\sigma_{i}=\mathcal{H}^{n}\left\llcorner\partial \Omega_{i}\right.$, $\omega_{i}$ denotes the harmonic measure of $\Omega_{i}$ either with pole at infinity or at $\frac{A-Q_{i}}{r_{i}}$, depending on whether $u$ is the Green's function with pole at infinite or with pole at $A$. Furthermore the corresponding Poisson kernel $h_{i}$ satisfies

$$
h_{i}(Q)=\frac{h\left(r_{i} Q+Q_{i}\right)}{\int_{B\left(Q_{i}, r_{i}\right)} h d \sigma} .
$$

Since $\log h \in \operatorname{VMO}(\partial \Omega)$, by including the term $\int_{B\left(Q_{i}, r_{i}\right)} h d \sigma$ in the denominator of the function $u_{i}$ defined in (3.2) we "remove the singularity" of the Poisson kernel of the limit domain. This is the "correct" type of blow up which allows us to connect the geometry of the limit domain to the analytic properties of its Green's function with pole at infinity.

Theorem 3.1. There exists a subsequence (which we relabel) satisfying $\Omega_{i} \longrightarrow \Omega_{\infty}$ in the Hausdorff distance sense, uniformly on compact sets,

$\partial \Omega_{i} \longrightarrow \partial \Omega_{\infty}$ in the Hausdorff distance sense, uniformly on compact sets, where $\Omega_{\infty}$ is an unbounded $4 \delta$-Reifenberg flat chord arc domain. Moreover

$$
u_{i} \longrightarrow u_{\infty} \text { uniformly on compact sets }
$$

and

$$
\left\{\begin{array}{rll}
\Delta u_{\infty}= & \text { in } \Omega_{\infty} \\
u_{\infty}=0 & \text { in } \partial \Omega_{\infty} \\
u_{\infty}>0 & \text { in } \Omega_{\infty} .
\end{array}\right.
$$

Furthermore

$$
\omega_{i} \rightarrow \omega_{\infty}
$$


and

$$
\sigma_{i} \rightarrow \sigma_{\infty}
$$

weakly as Radon measures. Here $\sigma_{\infty}=\mathcal{H}^{n} L_{-} \partial \Omega_{\infty}$, and $\omega_{\infty}$ denotes the harmonic measure of $\Omega_{\infty}$ with pole at infinity (corresponding to $u_{\infty}$ ). Moreover

$$
\sup _{Z \in \Omega_{\infty}}\left|\nabla u_{\infty}(Z)\right| \leq 1
$$

and

$$
h_{\infty}(Q)=\frac{d \omega_{\infty}}{d \sigma_{\infty}}(Q) \geq 1 \quad \text { for } \quad \mathcal{H}^{n} \text { - a.e. } \quad Q \in \partial \Omega_{\infty} .
$$

Since $\log h \in \operatorname{VMO}(\partial \Omega)$ the average $\int_{B(Q, r)} h d \sigma$ is close to the value of $\log h$ in a proportionally large subset of $B(Q, r) \cap \partial \Omega$. This remark allows us to conclude that (3.11) holds, which is crucial to the application, and which fails in general under just (3.6) and (3.7).

\section{Proof of the Main Theorem}

Let $K \subset \mathbb{R}^{n+1}$ be a compact set, and let

$$
l=\lim _{r \rightarrow 0} \sup _{Q \in \partial \Omega \cap K}\|\vec{n}\|_{*}(B(Q, r)),
$$

where

$$
\|\vec{n}\|_{*}(B(Q, r))=\sup _{0<s<r}\left(\int_{B(Q, s)}\left|\vec{n}-\overrightarrow{n_{Q, s}}\right|^{2} d \sigma\right)^{\frac{1}{2}}
$$

and $\overrightarrow{n_{Q, s}}=\int_{B(Q, s)} \vec{n} d \sigma$.

Our goal is to show that $l=0$. There exist sequences $\left\{Q_{i}\right\}_{i \geq 1} \subset \partial \Omega \cap K$, and $\left\{r_{i}\right\}_{i \geq 1} \subset \mathbb{R}$ such that $\lim _{i \rightarrow \infty} Q_{i}=Q_{\infty}, 0 \leq r_{i}, \lim _{i \rightarrow \infty} r_{i}=0$ and

$$
\lim _{i \rightarrow \infty}\left(\int_{B\left(Q_{i}, r_{i}\right)}\left|\vec{n}-\vec{n} Q_{i}, r_{i}\right|^{2} d \sigma\right)^{\frac{1}{2}}=l .
$$

We consider the blow up sequences $\Omega_{i}=r_{i}^{-1}\left(\Omega-Q_{i}\right), \partial \Omega_{i}=r_{i}^{-1}\left(\partial \Omega-Q_{i}\right)$, $u_{i}, \omega_{i}$ and $h_{i}$ associated with $Q_{i}$ and $r_{i}$ as in (3.2), (3.4) and (3.5). Then (3.6), (3.7), (3.8), (3.9), (3.10), (3.12), and (3.13), combined with Theorem 2.1 ensure that by passing to a subsequence (which we relabel), and modulo rotation we have that

$$
\begin{aligned}
\Omega_{i} \longrightarrow \mathbb{R}_{+}^{n+1} & \begin{array}{l}
\text { in the Hausdorff distance sense, } \\
\text { uniformly on compact sets, }
\end{array} \\
\partial \Omega_{i} \longrightarrow \mathbb{R}^{n} \times\{0\} & \begin{array}{l}
\text { in the Hausdorff distance sense } \\
\text { uniformly on compact sets, }
\end{array}
\end{aligned}
$$

and

$$
\sigma_{i}, \omega_{i} \rightarrow \mathcal{H}^{n}\left\llcorner\mathbb{R}^{n} \times\{0\}\right.
$$


Since $\chi_{\Omega_{i}} \rightarrow \chi_{\mathbb{R}_{+}^{n+1}}$ in $L_{\text {loc }}^{1}\left(\mathbb{R}^{n+1}\right)$, for $\varphi \in C_{c}^{\infty}\left(\mathbb{R}^{n+1}\right), \varphi \geq 0$ we have

$$
\lim _{i \rightarrow \infty} \int_{\Omega_{i}} \operatorname{div}\left(\varphi e_{n+1}\right)=\int_{\mathbb{R}_{+}^{n+1}} \operatorname{div}\left(\varphi e_{n+1}\right)
$$

If $\overrightarrow{n_{i}}$ denotes the inner unit normal to $\partial \Omega_{i}$ we have that

$$
\int_{\partial \Omega_{i}} \varphi\left\langle\overrightarrow{n_{i}}, e_{n+1}\right\rangle d \sigma_{i}=-\int_{\Omega_{i}} \operatorname{div}\left(\varphi e_{n+1}\right) .
$$

Therefore

$$
\lim _{i \rightarrow \infty} \int_{\partial \Omega_{i}} \varphi\left\langle\overrightarrow{n_{i}}, e_{n+1}\right\rangle d \sigma_{i}=\int_{\mathbb{R}^{n} \times\{0\}} \varphi d \mathcal{H}^{n}
$$

and hence

$$
\lim _{i \rightarrow \infty}\left\{\int_{\partial \Omega_{i}} \varphi d \sigma_{i}-\frac{1}{2} \int_{\partial \Omega_{i}} \varphi\left|\overrightarrow{n_{i}}-e_{n+1}\right|^{2} d \sigma_{i}\right\}=\int_{\mathbb{R}^{n} \times\{0\}} \varphi d \mathcal{H}^{n}
$$

Thus (3.11) yields

$$
\lim _{i \rightarrow \infty} \int_{\partial \Omega_{i}} \varphi\left|\overrightarrow{n_{i}}-e_{n+1}\right|^{2} d \sigma_{i}=0
$$

Letting $\varphi \geq \chi_{B(0,1)},(4.11)$ shows that

$$
\lim _{i \rightarrow \infty} \int_{B(0,1)}\left|\overrightarrow{n_{i}}-e_{n+1}\right|^{2} d \sigma_{i}=0 .
$$

Note that for $Q \in \partial \Omega_{i}, \overrightarrow{n_{i}}(Q)=\vec{n}\left(r_{i} Q+Q_{i}\right)$ where $\vec{n}$ denotes the inner unit normal to $\partial \Omega$. Furthermore

$$
\int_{B(0,1)}\left|\overrightarrow{n_{i}}-e_{n+1}\right|^{2} d \sigma_{i}=\int_{B\left(Q_{i}, r_{i}\right)}\left|\vec{n}-e_{n+1}\right|^{2} d \sigma
$$

Combining $(4.3),(4.12)$ and $(4.13)$ we conclude that $l=0$. In fact note that

$$
\begin{aligned}
l & =\lim _{i \rightarrow \infty}\left(\int_{B\left(Q_{i}, r_{i}\right)}\left|\vec{n}-\vec{n} Q_{i}, r_{i}\right|^{2} d \sigma\right)^{\frac{1}{2}} \\
& \leq 2 \lim _{i \rightarrow \infty}\left(\int_{B\left(Q_{i}, r_{i}\right)}\left|\vec{n}-e_{n+1}\right|^{2} d \sigma\right)^{\frac{1}{2}}=0 .
\end{aligned}
$$

\section{References}

[AC] H. W. Alt, L. A. Caffarelli, Existence and regularity for a minimum problem with free boundary, J. Reine Angew. Math. 325 (1981), 105-144.

[Du] P. Duren, The theory of $H^{p}$ spaces, Academic Press, New York, 1970.

[EG] L. C. Evans, R. F. Gariepy, Measure theory and fine properties of functions, Studies in Advanced Mathematics, CRC Press, Boca Raton, FL, 1992.

[J] D. Jerison, Regularity of the Poisson kernel and free boundary problems, Colloq. Math., 60/61 (1990), no. 2, 547-568. 
[JK1] D. Jerison, C. Kenig, Boundary behavior of harmonic functions in nontangentially accessible domains, Adv. in Math. 46 (1982), no. 1, 80-147.

[JK2] - The logarithm of the Poisson kernel of a $C^{1}$ domain has vanishing mean oscillation, Trans. Amer. Math. Soc. 273 (1982), no. 2, 781-794.

[KL] M. V. Keldysh, M. A. Lavrentiev, Sur la représentation conforme des domaines limités par des courbes rectifiables, Ann. Sci. École. Norm. Sup., 54 (1937), 1-38.

[KT1] C. Kenig, T. Toro, Free boundary regularity for harmonic measures and Poisson kernels, Ann. of Math. (2) 150 (1999), no. 2, 369-454.

[KT2] _ Poisson kernel characterization of Reifenberg flat chord arc domains, preprint.

[KT3] _ Harmonic measure on locally flat domains, Duke Math. J. 87 (1997), no. 3, $509-551$.

[KT4] - On the free boundary regularity theorem of Alt and Caffarelli, preprint.

[P] Ch. Pommerenke, On univalent functions, Bloch functions and VMOA, Math. Ann. 236 (1978), no. 3, 199-208.

5734 S. University Avenue, Department of Mathematics, University of Chicago, Chicago, IL 60637, U.S.A.

E-mail address: cek@math.uchicago.edu

Department of Mathematics, University of Washington, Box 354350, Seattle, WA 98195, U.S.A.

E-mail address: toro@math.washington.edu 